\title{
Risk factors for pertussis in adults and teenagers in England
}

\author{
A. WENSLEY ${ }^{1} \dagger$, G. J. HUGHES ${ }^{1} \dagger$, H. CAMPBELL ${ }^{2}$, G. AMIRTHALINGAM ${ }^{2}$, \\ N. ANDREWS ${ }^{3}, \mathrm{~N} \cdot \mathrm{YOUNG}^{4}$ AND L. COOLE ${ }^{1 *}$ \\ ${ }^{1}$ Field Epidemiology Service, National Infections Service, Public Health England, Leeds, UK \\ 2 Immunisation, Hepatitis and Blood Safety, Public Health England, London, UK \\ ${ }^{3}$ Statistics and Modelling Economics Department, Public Health England, London, UK \\ ${ }^{4}$ Public Health England South West, Exeter, UK
}

Received 31 May 2016; Final revision 15 November 2016; Accepted 15 November 2016; first published online 9 January 2017

\section{SUMMARY}

Pertussis is a vaccine-preventable respiratory infection caused by Bordetella pertussis which can be fatal in infants. Although high vaccine coverage led to prolonged disease control in England, a national outbreak of pertussis in 2011 led to the largest increase in over two decades, including a marked increase in cases aged $\geqslant 15$ years. A case-control study in four regions of England was undertaken to investigate risk factors for pertussis in adolescents and adults, specifically employment type and professional and household contact with children. Pertussis cases were laboratory-confirmed and aged $\geqslant 15$ years. Controls were recruited through general practitioner nomination. Demographic and risk factor information were collected using an online survey. Multivariable logistic regression was used to estimate independent associations with outcome. Two hundred and thirty-one cases and 190 controls were recruited. None of the four employment variables (social care, education, health sector, patient contact) were significantly associated with pertussis. Professional contact with children aged $<1$ year was associated with a significantly reduced odds of pertussis [odds ratio (OR) $0 \cdot 25,95 \%$ confidence interval (CI) $0 \cdot 08-0 \cdot 78$, $P=0 \cdot 017]$. Household contact with $\geqslant 1$ child aged $10-14$ years was associated with significantly increased odds of pertussis (OR 2.61, 95\% CI 1·47-4.64, $P=0 \cdot 001$ ). Occupational contact with very young children was associated with reduced odds of pertussis, probably due to immune boosting by low-level exposures to $B$. pertussis. Sharing a household with a young adolescent was a significant risk factor for pertussis in adults and older teenagers. The primary focus of the childhood pertussis vaccination programmes is to prevent infant disease. Although evidence is emerging that adolescent vaccination does not provide indirect protection to infants, our results highlight the importance of children aged 10-14 years in pertussis transmission to older adolescents and adults.

Key words: Pertussis, pertussis vaccine, risk factors, whooping cough.

\footnotetext{
* Author for correspondence: Dr L. Coole, Field Epidemiology Service, National Infections Service, Public Health England, Blenheim House, West One, Duncombe Street, Leeds LS1 4PL, UK.

(Email: louise.coole@phe.gov.uk).

$\dagger$ These authors contributed equally to this work.
}

\section{INTRODUCTION}

Pertussis (commonly known as whooping cough) is a vaccine-preventable bacterial respiratory infection caused by Bordetella pertussis which can be fatal in 
infants [1]. For older children and adults, symptoms are milder and often unrecognized as pertussis, but nonetheless associated with a considerable burden of illness [2]. About 25\% of infections in previously fully susceptible individuals will be asymptomatic and have been considered to be without an onwards risk of transmission [3].

The introduction of whole-cell pertussis (wP) vaccination in 1957 led to a rapid reduction in pertussis incidence in England and Wales [4]. However, after vaccine safety concerns in the 1970s and a marked decline in vaccine coverage, there followed a period of increased incidence that lasted until 1990, when confidence in vaccination was restored; vaccine coverage in England and Wales recovered to $92 \%$ by 1992 and has been sustained at that level or higher ever since [4].

In 2001, acellular pertussis (aP) vaccine was introduced into the routine childhood immunization programme as a preschool booster dose in England and Wales, and in 2004 aP vaccine replaced wP vaccine in the primary schedule [4]. There are different aP and wP vaccines available, of which certain formulations of each type appear equally efficacious against disease, although the aP vaccine is less reactogenic [5] but associated with more rapidly waning immunity [1]. After immune priming with multiple doses of aP vaccines, high levels of protection against disease are present for 4-12 years in children [6], after which the risk of pertussis significantly increases [7]. Consequently, the use of aP vaccines has been associated with a concomitant increase in pertussis in vaccinated adolescents $[8,9]$ and a general resurgence in disease in some countries [10]. In addition to waning immunity, a recent study using a baboon model of pertussis suggests that whereas wP-vaccinated individuals are protected against colonization, aP-vaccinated individuals, due to a qualitatively different immune response, are protected against disease but not against asymptomatic or mild infection with the potential for onward transmission [11].

High coverage with pertussis-containing vaccines resulted in a prolonged period of effective disease control in England. However, in late 2011 a national outbreak of pertussis began which resulted in the largest increase in cases seen in over two decades, peaking in 2012. Following increased infant disease and deaths during this outbreak, a UK pertussis vaccination programme for pregnant women was introduced from October 2012. This was later shown to have a high effectiveness for infants born to vaccinated mothers [12,13]. Prior to the 2011 outbreak, a trend for increasing cases of pertussis in those aged $\geqslant 15$ years in England had been observed [4], consistent both with increased case ascertainment in England [4] and a change in global epidemiology [14]. As the outbreak in England developed, a further marked increase in incidence rates in those aged $\geqslant 15$ years was observed [15].

In contrast to infants, where household contacts are the source for most cases [16], for adults, the main sources of pertussis are considered to be their children and work colleagues [17]. Outbreaks of pertussis in healthcare settings have involved a number of different transmission routes, including transmission of pertussis between healthcare workers (HCWs) [18], infection of HCWs through contact with patients with pertussis [19], and a combination of both [20]. Studies have suggested that $1-6 \%$ of paediatric HCWs develop asymptomatic infection with $B$. pertussis [21, 22], although seroprevalence may not differ from that of the general population [23]. During the 2011 outbreak, booster vaccination of HCWs was considered as a control measure in order to reduce transmission of pertussis to neonates and young infants [24], but, as it is not considered the optimal strategy for reducing the burden of infection for infants, was not implemented. Evidence from France and Australia also suggests that vaccine uptake by HCWs can be low [25, 26].

In early 2012, as part of the public health response to the outbreak in England, a national prospective case-control study of laboratory-confirmed pertussis in persons aged $\geqslant 15$ years was undertaken. The aim of this study was to investigate whether employment within different sectors, professional contact with children and young adults, and household contact with children and young adults were independent risk factors for pertussis for those aged $\geqslant 15$ years.

\section{METHODS}

\section{Study design}

A case-control study of persons aged $\geqslant 15$ years in England registered with a general practice (GP). Target recruitment was 250 cases and 500 matched controls (1:2 ratio of cases to controls) to provide $80 \%$ power (at $5 \%$ significance) to detect a minimum odds ratio (OR) of 2.4 with a $5 \%$ prevalence of exposure in controls. Formal ethical approval was not required as the study was undertaken as part of the public health response to an outbreak. 


\section{Case definition}

A case was defined as a person resident in one of four regions of England (Yorkshire and the Humber, South West, West Midlands, London) aged $\geqslant 15$ years with clinical signs or symptoms consistent with pertussis and either (1) a nasopharyngeal aspirate or per-nasal swab positive for $B$. pertussis by culture, (2) an anti-pertussis toxin $\mathrm{IgG}$ titre $>70 \mathrm{IU} / \mathrm{ml}$, or (3) a clinical specimen testing positive for $B$. pertussis by polymerase chain reaction. Cases with positive serology in the absence of isolation or detection of B. pertussis were excluded if they were known to have received vaccination for pertussis within the last year.

\section{Study participant recruitment}

Cases were recruited by local health protection teams within the four regions. Controls were recruited for each case according to GP, age group and sex. The GP of each case was asked to contact by post 10-15 suitable controls (each fifth name from the practice register of the same sex as the case and within the same 5-year age group). Each participating practice was provided with a set of pre-paid envelopes containing letters of invitation to participate which they were requested to send to the nominated controls. The GP was asked to exclude controls if the individual was assessed as inappropriate for inclusion or known to have previously had pertussis. If a GP declined to participate a neighbouring practice was selected and approached to nominate controls.

\section{Data collection}

Data were captured using an online questionnaire in SelectSurvey (https://selectsurvey.net). The questionnaire captured demographics, history of recent foreign travel (3 months prior to onset of illness for cases and interview date for controls), and selected questions designed to test three specific hypotheses related to the odds of having pertussis for individuals aged $\geqslant 15$ years: (1) employment within different sectors, (2) professional contact with children and young adults, and (3) household contact with children and young adults. Cases were interviewed by local health protection staff while controls were either interviewed by a member of the study team or self-completed using the online questionnaire. Each case plus associated controls was interviewed by the same interviewer wherever possible. All interviews were administered by local health protection and field epidemiology teams.

\section{Statistical analysis}

Univariable associations with outcome were calculated as unadjusted odds ratios (uOR). To explore confounding between two explanatory variables, Mantel-Haenszel odds ratios $\left(\mathrm{OR}_{\mathrm{MH}}\right)$ were calculated. Logistic regression was used to estimate adjusted odds ratios (aOR) for associations between explanatory variables and outcomes. Base variables of demographics (age quintiles, sex, region of residence) and recent foreign travel were included in all multivariable models irrespective of statistical significance. Five multivariable models were developed:

- M1: base variables only.

- M2: base variables + employment type variables.

- M3: base variables + professional contact with children/young adult variables.

- M4: base variables + household contact variables.

- M5: base variables + variables from all other categories.

For M1, two separate models were developed, specifying age either as a linear variable or using fractional polynomial transformations. The fit of these models was compared to the model where age was specified as quintiles using a likelihood ratio test (LRT). The most appropriate specification for age was then used for all subsequent models. For M2-4, a parsimony approach was taken for building multivariable models for each set of hypothesis testing variables. Firstly, a sub-model including all variables together (M2A, M3A, M4A) was constructed for each hypothesis in order to assess confounding and/or collinearity within each of the three variable sets. In order to determine the order variables were to be added for building the final parsimonious model for each hypothesis, variables were first added individually to the base variables (one model per variable; model set M2B, M3B, M4B). For construction of the final model for each hypothesis (M2C, M3C, M4C), variables were added to the base variables one at a time in order of increasing statistical significance based on the $P$ value from model set B. Variables were retained in the final model for each hypothesis if their inclusion significantly improved fit (LRT, $P<0 \cdot 05$ ). For M5, variables from all three hypotheses with a univariable association with outcome of $P<0 \cdot 2$ were considered for inclusion in the same model. All of these variables 
were added simultaneously to the base variables and then removed in order of descending $P$ value. The removal of each variable was assessed through a LRT comparing the reduced model to the previous one. The final model was reached when all variables additional to the base variables had an associated $P<0.05$. The fit of final models for M1-5 was assessed using the Hosmer-Lemeshow goodness-of-fit test [27]. All analysis was carried out in Stata v. 13.1 (StataCorp., USA).

\section{RESULTS}

\section{Study participants}

Study participants were recruited between 1 June and 31 October 2012. A total of 231 cases and 190 controls completed questionnaires and were eligible for inclusion in the study. As was expected for a case-control study of this nature, the response rate for potential controls was low $(6.8 \%$ in the one study region where data on response rates was available). For the one study region where data was available on participation from a subset of GPs contacted, 1/42 (2.4\%) of those practices declined to take part in control selection. The actual statistical power of the study was $80 \%$ to detect a minimum OR of 3.0 for $5 \%$ prevalence of exposure in controls. Descriptive characteristics of cases and controls are given in Table 1. Cases were on average slightly younger than controls (cases: mean age $43 \cdot 5$, range $15-87$ years; controls: mean age $50 \cdot 1$, range $15-85$ years). Data was missing only for three controls for region of residence.

\section{Statistical analysis}

Due to low levels of control recruitment, data were analysed using unconditional logistic regression with variables used for matching (age and sex) included in all models. Multivariable models were built in order to specifically test each of the three study hypotheses (Tables 2-4). Neither a linear specification of age nor a fractional polynomial transformation provided a significantly improved fit compared with age quintiles (both $P>0.05$ ).

\section{Associations between pertussis and employment within different sectors}

None of the four variables considered (employment in social care, employment in education, employment in the health sector, employment with direct patient contact) were significantly associated with pertussis either in a univariable analysis or after adjustment for other variables in a multivariable model (Table 2). The final model (M2C) provided reasonable fit to the data $\left[\chi^{2}(82, n=418)=94 \cdot 08, P=0 \cdot 171\right]$.

\section{Associations between pertussis and professional contact with young children and adults}

Two professional contact variables (contact with children aged $<1$ year, contact with preschool children) were significantly associated with outcome in a univariable analysis (Table 3). After adjustment within a multivariable model, only professional contact with children aged $<1$ year remained significant, with exposure associated with a reduced odds of pertussis [aOR 0.25, 95\% confidence interval (CI) 0.08$0 \cdot 78, P=0 \cdot 017]$. The final model (M3C) provided reasonable fit to the data $\left[\chi^{2}(69, n=418)=77 \cdot 39, P=\right.$ 0.229].

Study participants were provided with the option of selecting more than one age group of children they worked with, and of the 24 participants who indicated they worked with preschool children, $14(66 \cdot 7 \%)$ also indicated they worked with children aged $<1$ year. The significant univariable association between working with preschool children is explained by confounding with also working with children aged $<1$ year: after stratification by working with children aged $<1$ year there was no significant association between pertussis and working with preschool children $\left(\mathrm{OR}_{\mathrm{MH}}\right.$ $0 \cdot 68,95 \%$ CI $0 \cdot 19-2 \cdot 40, P=0 \cdot 370$ ).

\section{Associations between pertussis and household contacts with children and young adults}

Three household contact variables (household contact with $\geqslant 1$ child or young adult aged $0-21$ years, household contact with $\geqslant 1$ child aged $10-14$ years, household contact with $\geqslant 1$ child or adult aged 10-21 years) and a total household size of $\geqslant 3$ persons were significantly associated with outcome during a univariable analysis (Table 4). Given the overlap between age groups, substantial collinearity in prediction was observed. After considering each variable in a stepwise forward selection approach, only household contact with $\geqslant 1$ child aged $10-14$ years was included in the final model, with exposure associated with significantly increased odds of pertussis (aOR $2 \cdot 61,95 \%$ CI $1 \cdot 47-4 \cdot 64, P=0 \cdot 001)$. The final model 
Table 1. Characteristics of study participants: a case-control study of risk factors for pertussis in adults and teenagers in England

\begin{tabular}{|c|c|c|c|c|c|}
\hline \multirow[b]{2}{*}{$\begin{array}{l}\text { Risk factor } \\
\text { group }\end{array}$} & \multirow[b]{2}{*}{ Variable } & \multirow[b]{2}{*}{ Category* } & \multicolumn{3}{|c|}{ Number of participants (\%) } \\
\hline & & & $\begin{array}{l}\text { All } \\
(n=421)\end{array}$ & $\begin{array}{l}\text { Cases } \\
(n=231)\end{array}$ & $\begin{array}{l}\text { Controls } \\
(n=190)\end{array}$ \\
\hline \multirow{12}{*}{ Demographics } & \multirow{5}{*}{ Age quintiles $\dagger$} & Q1: 15-32 years & $89(21 \cdot 1)$ & $59(25 \cdot 5)$ & $30(15 \cdot 8)$ \\
\hline & & Q2: $33-43$ years & $85(20 \cdot 2)$ & $54(23 \cdot 4)$ & $31(16 \cdot 3)$ \\
\hline & & Q3: 44-51 years & $88(20 \cdot 9)$ & $51(22 \cdot 1)$ & $37(19 \cdot 5)$ \\
\hline & & Q4: 52-61 years & $75(17 \cdot 8)$ & $35(15 \cdot 2)$ & $40(21 \cdot 1)$ \\
\hline & & Q5: 62-87 years & $84(20 \cdot 0)$ & $32(13 \cdot 9)$ & $52(27 \cdot 4)$ \\
\hline & \multirow[t]{2}{*}{ Sex } & Male & $153(36 \cdot 3)$ & $97(50 \cdot 0)$ & $56(29 \cdot 5)$ \\
\hline & & Female & $268(63 \cdot 7)$ & $134(58 \cdot 0)$ & $134(70 \cdot 5)$ \\
\hline & \multirow[t]{5}{*}{ Region } & London & $24(5 \cdot 7)$ & $17(7 \cdot 4)$ & $7(3 \cdot 7)$ \\
\hline & & South West & $39(9 \cdot 3)$ & $28(12 \cdot 1)$ & $11(5 \cdot 8)$ \\
\hline & & West Midlands & $116(27 \cdot 6)$ & $68(29 \cdot 4)$ & $48(25 \cdot 3)$ \\
\hline & & Yorkshire \& the Humber & $239(56 \cdot 8)$ & $118(51 \cdot 1)$ & $121(63 \cdot 7)$ \\
\hline & & Missing & $3(0 \cdot 7)$ & 0 & $3(1 \cdot 6)$ \\
\hline Travel & Recent travel & Yes & $114(27 \cdot 1)$ & $57(24 \cdot 7)$ & $57(30 \cdot 0)$ \\
\hline \multirow{13}{*}{ Occupation: } & Social care & Yes & $16(3 \cdot 8)$ & $11(4 \cdot 8)$ & $5(2 \cdot 6)$ \\
\hline & Education & Yes & $84(20 \cdot 0)$ & $46(19 \cdot 9)$ & $38(20 \cdot 0)$ \\
\hline & \multirow[t]{2}{*}{ Health } & Any & $47(11 \cdot 2)$ & $26(11 \cdot 2)$ & $21(11 \cdot 1)$ \\
\hline & & Patient contact & $39(9 \cdot 3)$ & $23(10 \cdot 0)$ & $16(8 \cdot 4)$ \\
\hline & \multirow{9}{*}{$\begin{array}{l}\text { Professional contact with } \\
\text { children or young adults } \S\end{array}$} & $<1$ year & $18(4 \cdot 3)$ & $5(2 \cdot 2)$ & $13(6 \cdot 8)$ \\
\hline & & Preschool ( $<5$ years) & $24(5 \cdot 7)$ & $8(3 \cdot 5)$ & $16(8 \cdot 4)$ \\
\hline & & Primary school (5-11 years) & $41(9 \cdot 7)$ & $20(8 \cdot 7)$ & $21(11 \cdot 1)$ \\
\hline & & Young children $(<11$ years $)$ & $48(11 \cdot 4)$ & $22(9 \cdot 5)$ & $26(13 \cdot 7)$ \\
\hline & & Secondary school (11-16 years) & $42(10 \cdot 0)$ & $20(8 \cdot 7)$ & $22(11 \cdot 6)$ \\
\hline & & College (16-18 years) & $50(11.9)$ & $24(10 \cdot 4)$ & $26(13 \cdot 7)$ \\
\hline & & University (18-21 years) & $51(12 \cdot 1)$ & $25(10 \cdot 8)$ & $26(13 \cdot 7)$ \\
\hline & & Older children (11-21 years) & $77(18 \cdot 3)$ & $40(17 \cdot 3)$ & $37(19 \cdot 5)$ \\
\hline & & Any age & $102(24 \cdot 2)$ & $53(22 \cdot 9)$ & $49(25 \cdot 8)$ \\
\hline \multirow{11}{*}{$\begin{array}{c}\text { Household } \\
\text { contacts\| }\end{array}$} & \multirow[t]{9}{*}{$\geqslant 1$ child or young adult } & Any age $(0-21$ years $)$ & $206(48 \cdot 9)$ & $130(56 \cdot 3)$ & $76(40 \cdot 0)$ \\
\hline & & $<1$ year & $14(3 \cdot 3)$ & $11(4 \cdot 8)$ & $3(1 \cdot 6)$ \\
\hline & & $1-4$ years & $33(7 \cdot 8)$ & $17(7 \cdot 4)$ & $16(8 \cdot 4)$ \\
\hline & & 5-9 years & $60(14 \cdot 3)$ & $37(16 \cdot 0)$ & $23(12 \cdot 1)$ \\
\hline & & Young children ( $\leqslant 9$ years) & $88(20 \cdot 9)$ & $53(22 \cdot 9)$ & $35(18 \cdot 4)$ \\
\hline & & $10-14$ years & $91(21 \cdot 6)$ & $69(29 \cdot 9)$ & $22(11 \cdot 6)$ \\
\hline & & $15-19$ years & $91(21 \cdot 6)$ & $57(24 \cdot 7)$ & $34(17 \cdot 9)$ \\
\hline & & $20-21$ years & $20(4 \cdot 8)$ & $14(6 \cdot 0)$ & $6(3 \cdot 2)$ \\
\hline & & Older children (10-21 years) & $160(38 \cdot 0)$ & $110(47 \cdot 6)$ & $50(26 \cdot 3)$ \\
\hline & \multirow{2}{*}{ Household size } & $\leqslant 2$ & $195(46 \cdot 3)$ & $90(39 \cdot 0)$ & $105(55 \cdot 3)$ \\
\hline & & $\geqslant 3$ & $226(53 \cdot 7)$ & $141(61 \cdot 0)$ & $85(44 \cdot 7)$ \\
\hline
\end{tabular}

* Categories of missing data are only shown were $\geqslant 1$ case had missing data.

$\dagger$ Q1-Q5, quintiles 1-5.

$¥$ Studying or working in each specific area.

$\S$ Working with children from specific age groups.

|| Individuals living within the same household.

(M4C) provided reasonable fit to the data $\left[\chi^{2}(89, n=\right.$ 418) $=98 \cdot 19, P=0 \cdot 237]$.

Total household size was no longer significantly associated with pertussis after adjusting for other household variables (model M4A), and was not included in the final model. The significant univariable association for total household size can be explained by confounding with household contact with $\geqslant 1$ child aged 10-14 years: after stratification by household contact with $\geqslant 1$ child aged 10-14 years there was no significant association between pertussis and total household size $\left(\mathrm{OR}_{\mathrm{MH}} 1 \cdot 41,95 \% \mathrm{CI} 0 \cdot 91-2 \cdot 16, P=0 \cdot 124\right)$. 
Table 2. Crude and adjusted associations between pertussis and occupational risk factors in adults and teenagers in England

\begin{tabular}{|c|c|c|c|c|c|c|c|c|c|c|c|c|c|}
\hline \multirow{3}{*}{$\begin{array}{l}\text { Risk factor } \\
\text { group }\end{array}$} & \multirow[b]{3}{*}{ Variable } & \multirow[b]{3}{*}{ Category } & & & \multicolumn{9}{|c|}{ Multivariable models } \\
\hline & & & \multicolumn{2}{|c|}{ Univariable associations } & \multicolumn{2}{|l|}{ M1 } & \multicolumn{2}{|l|}{$\mathrm{M} 2 \mathrm{~A}^{*}$} & \multicolumn{2}{|l|}{$\mathrm{M} 2 \mathrm{~B} \dagger$} & \multicolumn{3}{|l|}{$\mathrm{M} 2 \mathrm{C}$} \\
\hline & & & uOR $(95 \% \mathrm{CI})$ & $P$ & $\mathrm{aOR}(95 \% \mathrm{CI})$ & $P$ & $\mathrm{aOR}(95 \% \mathrm{CI})$ & $P$ & $\mathrm{aOR}(95 \% \mathrm{CI})$ & $P$ & $\chi^{2}(P) \S$ & $\mathrm{aOR}(95 \% \mathrm{CI})$ & $P$ \\
\hline \multirow[t]{11}{*}{ Demographics } & \multirow{5}{*}{$\begin{array}{l}\text { Age } \\
\text { quintiles }\end{array}$} & Q1: $15-32$ years & $3 \cdot 20(1 \cdot 82-5 \cdot 95)$ & $<0 \cdot 001$ & $3 \cdot 56(1 \cdot 86-6 \cdot 79)$ & $<0 \cdot 001$ & $3 \cdot 83(1.93-7 \cdot 61)$ & $<0 \cdot 001$ & - & - & - & $3.83(1.93-7 \cdot 61)$ & $<0.001$ \\
\hline & & Q2: $33-43$ years & $2 \cdot 83(1 \cdot 52-5 \cdot 28)$ & $0 \cdot 001$ & $3 \cdot 23(1 \cdot 68-6 \cdot 22)$ & $<0 \cdot 001$ & $3 \cdot 35(1 \cdot 73-6 \cdot 49)$ & $<0 \cdot 001$ & - & - & - & $3 \cdot 35(1 \cdot 73-6 \cdot 49)$ & $<0.001$ \\
\hline & & Q3: 44-51 years & $2 \cdot 24(1 \cdot 22-4 \cdot 13)$ & $0 \cdot 010$ & $2 \cdot 38(1 \cdot 25-4 \cdot 52)$ & $0 \cdot 008$ & $2 \cdot 37(1 \cdot 22-4 \cdot 60)$ & $0 \cdot 011$ & - & - & - & $2 \cdot 37(1 \cdot 22-4 \cdot 60)$ & $0 \cdot 011$ \\
\hline & & Q4: 52-61 years & $1 \cdot 42(0 \cdot 76-2 \cdot 68)$ & $0 \cdot 275$ & $1 \cdot 54(0 \cdot 80-2 \cdot 98)$ & $0 \cdot 204$ & $1 \cdot 48(0 \cdot 76-2 \cdot 90)$ & $0 \cdot 248$ & - & - & - & $1 \cdot 48(0 \cdot 76-2 \cdot 90)$ & $0 \cdot 248$ \\
\hline & & Q5: 62-87 years & Ref. & - & Ref. & - & Ref. & - & - & - & - & Ref. & - \\
\hline & \multirow[t]{2}{*}{ Sex } & Male & $1 \cdot 73(1 \cdot 15-2 \cdot 60)$ & $0 \cdot 008$ & $1 \cdot 84(1 \cdot 19-2 \cdot 83)$ & $0 \cdot 006$ & $1 \cdot 86(1 \cdot 19-2 \cdot 91)$ & $0 \cdot 006$ & - & - & - & $1 \cdot 86(1 \cdot 19-2 \cdot 91)$ & $0 \cdot 006$ \\
\hline & & Female & Ref. & - & Ref. & - & Ref. & - & - & - & - & Ref. & - \\
\hline & \multirow[t]{4}{*}{ Region } & London & $2 \cdot 49(1 \cdot 00-6 \cdot 22)$ & $0 \cdot 051$ & $1 \cdot 98(0 \cdot 76-5 \cdot 11)$ & $0 \cdot 160$ & $2 \cdot 04(0 \cdot 78-5 \cdot 34)$ & $0 \cdot 145$ & - & - & - & $2 \cdot 04(0 \cdot 78-5 \cdot 34)$ & $0 \cdot 145$ \\
\hline & & South West & $2 \cdot 61(1 \cdot 24-5 \cdot 48)$ & $0 \cdot 011$ & $3 \cdot 14(1 \cdot 43-6 \cdot 94)$ & $0 \cdot 005$ & $3 \cdot 17(1 \cdot 43-7 \cdot 04)$ & $0 \cdot 005$ & - & - & - & $3 \cdot 17(1 \cdot 43-7 \cdot 04)$ & $0 \cdot 005$ \\
\hline & & West Midlands & $1 \cdot 45(0 \cdot 93-2 \cdot 27)$ & $0 \cdot 102$ & $1 \cdot 42(0 \cdot 89-2 \cdot 26)$ & $0 \cdot 141$ & $1 \cdot 41(0 \cdot 88-2 \cdot 25)$ & $0 \cdot 154$ & - & - & - & $1 \cdot 41(0 \cdot 88-2 \cdot 25)$ & $0 \cdot 154$ \\
\hline & & $\begin{array}{l}\text { Yorkshire \& } \\
\text { the Humber }\end{array}$ & Ref. & - & Ref. & - & Ref. & - & - & - & - & Ref. & - \\
\hline Travel & $\begin{array}{r}\text { Recent } \\
\text { travel }\end{array}$ & Yes & $0 \cdot 76(0 \cdot 50-1 \cdot 17)$ & $0 \cdot 222$ & $0 \cdot 67(0 \cdot 42-1 \cdot 07)$ & $0 \cdot 094$ & $0 \cdot 67(0 \cdot 41-1 \cdot 07)$ & $0 \cdot 091$ & - & - & - & $0 \cdot 67(0 \cdot 41-1 \cdot 07)$ & $0 \cdot 091$ \\
\hline \multirow[t]{4}{*}{ Occupation } & Social care & Yes & $1 \cdot 85(0 \cdot 63-5 \cdot 42)$ & $0 \cdot 262$ & - & - & $2 \cdot 36(0 \cdot 75-7 \cdot 39)$ & $0 \cdot 141$ & $2 \cdot 46(0 \cdot 79-7 \cdot 63)$ & $0 \cdot 119$ & $2 \cdot 61(0 \cdot 106)$ & not included & - \\
\hline & Education & Yes & $0 \cdot 99(0 \cdot 62-1 \cdot 61)$ & $0 \cdot 982$ & - & - & $0 \cdot 82(0 \cdot 49-1 \cdot 42)$ & $0 \cdot 485$ & $0 \cdot 80(0 \cdot 47-1 \cdot 38)$ & $0 \cdot 424$ & $0 \cdot 64(0 \cdot 424)$ & not included & - \\
\hline & Health & Yes & $1.02(0.55-1.88)$ & 0.948 & - & - & $0 \cdot 59(0 \cdot 13-2 \cdot 65)$ & $0 \cdot 491$ & $1 \cdot 20(0 \cdot 62-2 \cdot 34)$ & 0.591 & $0.29(0.590)$ & not included & - \\
\hline & $\begin{array}{l}\text { Patient } \\
\text { contact }\end{array}$ & Yes & $1 \cdot 20(0 \cdot 62-2 \cdot 35)$ & $0 \cdot 589$ & - & - & $2 \cdot 18(0 \cdot 43-11 \cdot 14)$ & $0 \cdot 349$ & $1.44(0.69-2.99)$ & $0 \cdot 328$ & $0.97(0 \cdot 324)$ & not included & - \\
\hline
\end{tabular}

uOR, Unadjusted odds ratio; CI, confidence interval; aOR, adjusted odds ratio; Q1-Q5, quintiles 1-5; Ref., reference group.

Values in bold indicate statistical significance $(P<0 \cdot 05)$

* All occupation variables added simultaneously to M1.

$\uparrow$ A separate model produced for each occupation variable added to M1.

\$ Variables added to M1 in order of increasing statistical significance according to $P$ value from M2B, variables retained only if the lead to a statistically significant improvement in fit.

$\S \chi^{2}$ and associated $P$ value of the likelihood ratio test of improved fit compared to M1. 
Table 3. Crude and adjusted associations between pertussis and professional contact with children and young adults in adults and teenagers in England

\begin{tabular}{|c|c|c|c|c|c|c|c|c|c|c|c|c|c|}
\hline \multirow{3}{*}{$\begin{array}{l}\text { Risk factor } \\
\text { group }\end{array}$} & \multirow[b]{3}{*}{ Variable } & \multirow[b]{3}{*}{ Category } & & & \multicolumn{9}{|c|}{ Multivariable models } \\
\hline & & & \multicolumn{2}{|c|}{ Univariable associations } & \multicolumn{2}{|l|}{ M1 } & \multicolumn{2}{|l|}{ M3A* } & \multicolumn{2}{|l|}{$\mathrm{M} 3 \mathrm{~B} \dagger$} & \multicolumn{3}{|l|}{ M3C $\ddagger$} \\
\hline & & & uOR $(95 \%$ CI $)$ & $P$ & $\mathrm{aOR}(95 \% \mathrm{CI})$ & $P$ & $\mathrm{aOR}(95 \% \mathrm{CI})$ & $P$ & $\mathrm{aOR}(95 \% \mathrm{CI})$ & $P$ & $\chi^{2}(P) \S$ & aOR $(95 \% \mathrm{CI})$ & $P$ \\
\hline \multirow[t]{11}{*}{ Demographics } & \multirow{5}{*}{$\begin{array}{l}\text { Age } \\
\text { quintiles }\end{array}$} & Q1: 15-32 years & $3 \cdot 20(1 \cdot 82-5 \cdot 95)$ & $<0.001$ & $3 \cdot 56(1 \cdot 86-6 \cdot 79)$ & $<0.001$ & $4 \cdot 06(2 \cdot 06-8 \cdot 00)$ & $<0 \cdot 001$ & - & - & - & $4 \cdot 03(2 \cdot 08-7 \cdot 80)$ & $<0 \cdot 001$ \\
\hline & & Q2: 33-43 years & $2 \cdot 83(1 \cdot 52-5 \cdot 28)$ & $0 \cdot 001$ & $3 \cdot 23(1 \cdot 68-6 \cdot 22)$ & $<0.001$ & $3 \cdot 39(1 \cdot 73-6 \cdot 62)$ & $<0.001$ & - & - & - & $3 \cdot 39(1 \cdot 76-6 \cdot 52)$ & $<0.001$ \\
\hline & & Q3: 44-51 years & $2 \cdot 24(1 \cdot 22-4 \cdot 13)$ & $0 \cdot 010$ & $2 \cdot 38(1 \cdot 25-4 \cdot 52)$ & $0 \cdot 008$ & $2 \cdot 55(1 \cdot 30-5 \cdot 01)$ & $0 \cdot 007$ & - & - & - & $2 \cdot 57(1 \cdot 34-4.91)$ & $0 \cdot 004$ \\
\hline & & Q4: 52-61 years & $1.42(0 \cdot 76-2 \cdot 68)$ & $0 \cdot 278$ & $1.54(0 \cdot 80-2 \cdot 98)$ & $0 \cdot 204$ & $1 \cdot 59(0 \cdot 81-3 \cdot 12)$ & $0 \cdot 181$ & - & - & - & $1 \cdot 61(0 \cdot 83-3 \cdot 12)$ & $0 \cdot 163$ \\
\hline & & Q5: 62-87 years & Ref. & - & Ref. & - & Ref. & - & - & - & - & Ref. & - \\
\hline & \multirow[t]{2}{*}{ Sex } & Male & $1 \cdot 73(1 \cdot 15-2 \cdot 60)$ & $0 \cdot 008$ & $1 \cdot 84(1 \cdot 19-2 \cdot 83)$ & $0 \cdot 006$ & $1 \cdot 75(1 \cdot 12-2 \cdot 75)$ & $0 \cdot 014$ & - & - & - & $1 \cdot 73(1 \cdot 11-2 \cdot 80)$ & $0 \cdot 015$ \\
\hline & & Female & Ref. & - & Ref. & - & Ref. & - & - & - & - & Ref. & - \\
\hline & \multirow[t]{4}{*}{ Region } & London & $2 \cdot 49(1 \cdot 00-6 \cdot 22)$ & $0 \cdot 051$ & $1.98(0 \cdot 76-5 \cdot 11)$ & $0 \cdot 160$ & $2 \cdot 03(0 \cdot 77-5 \cdot 34)$ & $0 \cdot 153$ & - & - & - & $1.95(0 \cdot 74-5 \cdot 13)$ & $0 \cdot 174$ \\
\hline & & South West & $2 \cdot 61(1 \cdot 24-5 \cdot 48)$ & $0 \cdot 011$ & $3 \cdot 14(1 \cdot 43-6 \cdot 94)$ & $0 \cdot 005$ & $3 \cdot 35(1 \cdot 47-7 \cdot 65)$ & $0 \cdot 004$ & - & - & - & $3 \cdot 18(1 \cdot 44-7 \cdot 04)$ & $0 \cdot 004$ \\
\hline & & West Midlands & $1 \cdot 45(0 \cdot 93-2 \cdot 27)$ & $0 \cdot 107$ & $1 \cdot 42(0 \cdot 89-2 \cdot 26)$ & $0 \cdot 141$ & $1 \cdot 38(0 \cdot 86-2 \cdot 24)$ & $0 \cdot 185$ & - & - & - & $1 \cdot 32(0 \cdot 83-2 \cdot 12)$ & $0 \cdot 242$ \\
\hline & & $\begin{array}{l}\text { Yorkshire \& } \\
\text { the Humber }\end{array}$ & Ref. & - & Ref. & - & Ref. & - & - & - & - & Ref. & - \\
\hline Travel & $\begin{array}{r}\text { Recent } \\
\text { travel }\end{array}$ & Yes & $0 \cdot 76(0 \cdot 50-1 \cdot 17)$ & $0 \cdot 222$ & $0 \cdot 67(0 \cdot 41-1 \cdot 07)$ & $0 \cdot 094$ & $0 \cdot 68(0 \cdot 42-1 \cdot 10)$ & $0 \cdot 115$ & - & - & - & $0 \cdot 70(0 \cdot 43-1 \cdot 11)$ & $0 \cdot 130$ \\
\hline \multirow{9}{*}{\multicolumn{2}{|c|}{$\begin{array}{l}\text { Professional contact with } \\
\text { children or young adults }\end{array}$}} & $<1$ year & $0 \cdot 30(0 \cdot 11-0 \cdot 86)$ & $0 \cdot 025$ & - & - & $0 \cdot 22(0 \cdot 04-1 \cdot 23)$ & $0 \cdot 085$ & $0 \cdot 26(0 \cdot 08-0 \cdot 78)$ & $0 \cdot 017$ & $6 \cdot 42(0 \cdot 011)$ & $0 \cdot 25(0 \cdot 08-0 \cdot 78)$ & $0 \cdot 017$ \\
\hline & & $\begin{array}{l}\text { Preschool } \\
\text { (<5 years) }\end{array}$ & $0 \cdot 39(0 \cdot 16-0 \cdot 93)$ & $0 \cdot 034$ & - & - & $1 \cdot 21(0 \cdot 22-6 \cdot 61)$ & $0 \cdot 828$ & $0 \cdot 46(0 \cdot 18-1 \cdot 15)$ & $0 \cdot 095$ & $0 \cdot 12(0 \cdot 725)$ & not included & - \\
\hline & & $\begin{array}{l}\text { Primary school } \\
\text { (5-11 years) }\end{array}$ & $0 \cdot 76(0 \cdot 40-1 \cdot 45)$ & $0 \cdot 411$ & - & - & $2 \cdot 67(0 \cdot 34-20 \cdot 92)$ & $0 \cdot 350$ & $0 \cdot 90(0 \cdot 44-1 \cdot 81)$ & $0 \cdot 759$ & $1 \cdot 05(0 \cdot 305)$ & not included & - \\
\hline & & $\begin{array}{l}\text { Young children } \\
(<11 \text { years })\end{array}$ & $0 \cdot 66(0 \cdot 36-1 \cdot 21)$ & $0 \cdot 183$ & - & - & $0 \cdot 56(0 \cdot 03-9 \cdot 81)$ & $0 \cdot 691$ & $0 \cdot 74(0 \cdot 38-1 \cdot 43)$ & $0 \cdot 366$ & $0.49(0 \cdot 484)$ & not included & - \\
\hline & & $\begin{array}{l}\text { Secondary school } \\
\text { (11-16 years) }\end{array}$ & $0 \cdot 72(0 \cdot 35-1 \cdot 37)$ & $0 \cdot 321$ & - & - & $0 \cdot 99(0 \cdot 19-5 \cdot 11)$ & 0.988 & $0.78(0.39-1.53)$ & $0 \cdot 465$ & $0 \cdot 15(0 \cdot 701)$ & not included & - \\
\hline & & $\begin{array}{l}\text { College } \\
\text { (16-18 years) }\end{array}$ & $0 \cdot 73(0 \cdot 40-1 \cdot 32)$ & $0 \cdot 300$ & - & - & $0 \cdot 72(0 \cdot 24-2 \cdot 12)$ & $0 \cdot 551$ & $0 \cdot 71(0 \cdot 38-1 \cdot 33)$ & $0 \cdot 289$ & $0.09(0.760)$ & not included & - \\
\hline & & $\begin{array}{l}\text { University } \\
\text { (18-21 years) }\end{array}$ & $0 \cdot 77(0 \cdot 43-1 \cdot 38)$ & $0 \cdot 371$ & - & - & $0 \cdot 73(0 \cdot 18-2 \cdot 96)$ & 0.663 & $0 \cdot 72(0 \cdot 38-1 \cdot 33)$ & $0 \cdot 294$ & $0 \cdot 11(0 \cdot 739)$ & not included & - \\
\hline & & $\begin{array}{r}\text { Older children } \\
\text { (11-21 years) }\end{array}$ & $0 \cdot 87(0 \cdot 53-1 \cdot 42)$ & $0 \cdot 569$ & & - & $1 \cdot 51(0 \cdot 11-21 \cdot 50)$ & $0 \cdot 722$ & $0 \cdot 84(0 \cdot 49-1 \cdot 42)$ & 0.509 & $0.01(0.999)$ & not included & - \\
\hline & & Any age & $0 \cdot 86(0 \cdot 55-1 \cdot 34)$ & $0 \cdot 498$ & - & - & $0 \cdot 93(0 \cdot 14-6 \cdot 19)$ & 0.939 & $0 \cdot 84(0 \cdot 52-1 \cdot 38)$ & $0 \cdot 104$ & $0.04(0.847$ & not included & - \\
\hline
\end{tabular}

uOR, Unadjusted odds ratio; CI, confidence interval; aOR, adjusted odds ratio; Q1-Q5, quintiles 1-5; Ref., reference group.

Values in bold indicate statistical significance $(P<0 \cdot 05)$.

* All professional contact with children or young adult variables added simultaneously to M1.

$\dagger$ A separate model produced for each professional contact with children or young adult variable added to M1.

$\$$ Variables added to $\mathrm{M} 1$ in order of increasing statistical significance according to $P$ value from M3B, variables retained only if the lead to a statistically significant improvement in fit.

$\S \chi^{2}$ and associated $P$ value of the likelihood ratio test of improved fit compared to M1. 
Table 4. Crude and adjusted associations between pertussis and household contact in adults and teenagers in England

\begin{tabular}{|c|c|c|c|c|c|c|c|c|c|c|c|c|c|}
\hline \multirow{3}{*}{$\begin{array}{l}\text { Risk factor } \\
\text { group }\end{array}$} & \multirow[b]{3}{*}{ Variable } & \multirow[b]{3}{*}{ Category } & & & \multicolumn{9}{|c|}{ Multivariable models } \\
\hline & & & \multicolumn{2}{|c|}{ Univariable associations } & \multicolumn{2}{|l|}{ M1 } & \multicolumn{2}{|l|}{ M4A* } & \multicolumn{2}{|l|}{$\mathrm{M} 4 \mathrm{~B} \dagger$} & \multicolumn{3}{|l|}{ M4C } \\
\hline & & & uOR $(95 \% \mathrm{CI})$ & $P$ & $\mathrm{aOR}(95 \% \mathrm{CI})$ & $P$ & $\mathrm{aOR}(95 \% \mathrm{CI})$ & $P$ & $\mathrm{aOR}(95 \% \mathrm{CI})$ & $p$ & $\chi^{2}(P) \S$ & $\mathrm{aOR}(95 \% \mathrm{CI})$ & $P$ \\
\hline \multirow[t]{11}{*}{ Demographics } & \multirow{5}{*}{$\begin{array}{l}\text { Age } \\
\text { quintiles }\end{array}$} & Q1: $15-32$ years & $3 \cdot 20(1 \cdot 82-5 \cdot 95)$ & $<0 \cdot 001$ & $3 \cdot 56(1 \cdot 86-6 \cdot 79)$ & $<0 \cdot 001$ & $2 \cdot 79(1 \cdot 36-5 \cdot 74)$ & $0 \cdot 005$ & - & - & - & $2 \cdot 92(1 \cdot 51-5 \cdot 65)$ & $0 \cdot 001$ \\
\hline & & Q2: $33-43$ years & $2 \cdot 83(1 \cdot 52-5 \cdot 28)$ & $0 \cdot 001$ & $3 \cdot 23(1 \cdot 68-6 \cdot 22)$ & $<0.001$ & $2 \cdot 45(1 \cdot 09-5 \cdot 49)$ & $0 \cdot 029$ & - & - & - & $2 \cdot 33(1 \cdot 17-4 \cdot 63)$ & $0 \cdot 016$ \\
\hline & & Q3: 44-51 years & $2 \cdot 24(1 \cdot 22-4 \cdot 13)$ & $0 \cdot 010$ & $2 \cdot 38(1 \cdot 25-4 \cdot 52)$ & $0 \cdot 008$ & $1 \cdot 63(0 \cdot 78-3 \cdot 41)$ & $0 \cdot 191$ & - & - & - & $1 \cdot 70(0 \cdot 86-3 \cdot 33)$ & $0 \cdot 125$ \\
\hline & & Q4: 52-61 years & $1 \cdot 42(0 \cdot 76-2 \cdot 68)$ & $0 \cdot 278$ & $1.54(0 \cdot 80-2.98)$ & $0 \cdot 204$ & $1 \cdot 35(0 \cdot 68-2 \cdot 71)$ & $0 \cdot 394$ & - & - & - & $1 \cdot 40(0 \cdot 72-2 \cdot 72)$ & $0 \cdot 326$ \\
\hline & & Q5: 62-87 years & Ref. & - & Ref. & - & Ref. & - & - & - & - & Ref. & - \\
\hline & \multirow[t]{2}{*}{ Sex } & Male & $1 \cdot 73(1 \cdot 15-2 \cdot 60)$ & $0 \cdot 008$ & $1 \cdot 84(1 \cdot 19-2 \cdot 83)$ & $0 \cdot 006$ & $1 \cdot 69(1 \cdot 08-2 \cdot 66)$ & $0 \cdot 022$ & - & - & - & $1 \cdot 81(1 \cdot 66-2 \cdot 81)$ & $0 \cdot 008$ \\
\hline & & Female & Ref. & - & Ref. & - & Ref. & - & - & - & - & Ref. & - \\
\hline & \multirow[t]{4}{*}{ Region } & London & $2 \cdot 49(1 \cdot 00-6 \cdot 22)$ & $0 \cdot 051$ & $1 \cdot 98(0 \cdot 76-5 \cdot 11)$ & $0 \cdot 160$ & $2 \cdot 08(0 \cdot 78-5 \cdot 53)$ & $0 \cdot 142$ & - & - & - & $2 \cdot 12(0 \cdot 81-5 \cdot 55)$ & $0 \cdot 126$ \\
\hline & & South West & $2.61(1.24-5.48)$ & $0 \cdot 011$ & $3 \cdot 14(1 \cdot 43-6 \cdot 94)$ & $0 \cdot 005$ & $3 \cdot 05(1 \cdot 36-6 \cdot 83)$ & $0 \cdot 007$ & - & - & - & $3 \cdot 05(1 \cdot 37-6 \cdot 79)$ & 0.006 \\
\hline & & West Midlands & $1.45(0.93-2 \cdot 27)$ & $0 \cdot 107$ & $1 \cdot 42(0 \cdot 89-2 \cdot 26)$ & $0 \cdot 141$ & $1.53(0.94-2 \cdot 47)$ & $0 \cdot 084$ & - & - & - & $1 \cdot 46(0 \cdot 91-2 \cdot 34)$ & $0 \cdot 117$ \\
\hline & & $\begin{array}{l}\text { Yorkshire \& } \\
\text { the Humber }\end{array}$ & Ref. & - & Ref. & - & Ref. & - & - & - & - & Ref. & - \\
\hline Travel & Recent travel & Yes & $0 \cdot 76(0 \cdot 50-1 \cdot 17)$ & $0 \cdot 222$ & $0 \cdot 67(0 \cdot 41-1 \cdot 07)$ & $0 \cdot 094$ & $0 \cdot 71(0 \cdot 44-1 \cdot 15)$ & $0 \cdot 166$ & - & - & - & $0 \cdot 66(0 \cdot 41-1 \cdot 06)$ & $0 \cdot 085$ \\
\hline \multirow{11}{*}{$\begin{array}{l}\text { Household } \\
\text { contacts }\end{array}$} & \multirow{9}{*}{$\begin{array}{l}\geqslant 1 \text { child or } \\
\text { young adult }\end{array}$} & $<1$ year & $3 \cdot 12(0 \cdot 86-11 \cdot 34)$ & $0 \cdot 084$ & - & - & $8 \cdot 17(0 \cdot 99-67 \cdot 36)$ & $0 \cdot 051$ & $2 \cdot 72(0 \cdot 57-12 \cdot 97)$ & $0 \cdot 209$ & $2 \cdot 20(0 \cdot 14)$ & not included & - \\
\hline & & $1-4$ years & $0 \cdot 86(0 \cdot 42-1 \cdot 76)$ & 0.687 & - & - & $1 \cdot 34(0 \cdot 33-5 \cdot 46)$ & $0 \cdot 682$ & $0 \cdot 66(0 \cdot 29-1 \cdot 48)$ & $0 \cdot 308$ & $0.96(0.327)$ & not included & - \\
\hline & & $5-9$ years & $1 \cdot 38(0 \cdot 82-2 \cdot 13)$ & $0 \cdot 256$ & - & - & $2 \cdot 48(0 \cdot 47-13 \cdot 12)$ & $0 \cdot 287$ & $0 \cdot 96(0 \cdot 50-1 \cdot 86)$ & $0 \cdot 913$ & $0 \cdot 14(0 \cdot 704)$ & not included & - \\
\hline & & $\begin{array}{l}\text { Young children } \\
\text { ( } \leqslant 9 \text { years) }\end{array}$ & $1 \cdot 32(0 \cdot 82-2 \cdot 13)$ & $0 \cdot 257$ & - & - & $0 \cdot 46(0 \cdot 07-2 \cdot 98)$ & $0 \cdot 416$ & $0 \cdot 87(0 \cdot 48-1 \cdot 60)$ & $0 \cdot 646$ & $0 \cdot 54(0 \cdot 461)$ & not included & - \\
\hline & & $10-14$ years & $3 \cdot 25(1.92-5 \cdot 50)$ & $<0 \cdot 001$ & - & - & $1.90(0.74-4.87)$ & $0 \cdot 180$ & $2 \cdot 61(1 \cdot 47-4 \cdot 64)$ & $0 \cdot 001$ & $11 \cdot 43(<0 \cdot 001)$ & $2 \cdot 61(1 \cdot 47-4 \cdot 64)$ & $0 \cdot 001$ \\
\hline & & 15-19 years & $1 \cdot 50(0 \cdot 93-2 \cdot 42)$ & $0 \cdot 094$ & - & - & $0 \cdot 91(0 \cdot 35-2 \cdot 36)$ & $0 \cdot 842$ & $1 \cdot 34(0 \cdot 79-2 \cdot 28)$ & $0 \cdot 272$ & $0 \cdot 74(0.390)$ & not included & - \\
\hline & & 20-21 years & $1.98(0 \cdot 75-5 \cdot 25)$ & $0 \cdot 171$ & - & - & $1 \cdot 22(0 \cdot 37-3 \cdot 98)$ & $0 \cdot 744$ & $1 \cdot 41(0 \cdot 50-3 \cdot 94)$ & $0 \cdot 514$ & $0 \cdot 61(0.433)$ & not included & - \\
\hline & & $\begin{array}{l}\text { Older children } \\
(10-21 \text { years })\end{array}$ & $2 \cdot 55(1 \cdot 68-3 \cdot 85)$ & $<0 \cdot 001$ & - & - & $2 \cdot 71(0 \cdot 65-11 \cdot 32)$ & $0 \cdot 173$ & $2 \cdot 09(1 \cdot 30-3 \cdot 36)$ & $0 \cdot 002$ & $1 \cdot 63(0 \cdot 201)$ & not included & - \\
\hline & & $\begin{array}{l}\text { Any age } \\
(0-21 \text { years })\end{array}$ & $1.93(1 \cdot 31-2 \cdot 85)$ & $0 \cdot 001$ & - & - & $0 \cdot 47(0 \cdot 13-1 \cdot 71)$ & $0 \cdot 253$ & $1 \cdot 49(0 \cdot 92-2 \cdot 43)$ & $0 \cdot 107$ & $0 \cdot 02(0 \cdot 902)$ & not included & - \\
\hline & \multirow{2}{*}{$\begin{array}{l}\text { Household } \\
\text { size }\end{array}$} & $\leqslant 2$ & Ref. & - & - & - & Ref. & - & Ref. & - & - & - & - \\
\hline & & $\geqslant 3$ & $1.94(1 \cdot 31-2 \cdot 86)$ & $\mathbf{0} \cdot 001$ & - & - & $1 \cdot 09(0 \cdot 55-2 \cdot 14)$ & $0 \cdot 805$ & $1 \cdot 40(0 \cdot 87-2 \cdot 25)$ & $0 \cdot 160$ & $0 \cdot 16$ & not included & - \\
\hline
\end{tabular}

uOR, Unadjusted odds ratio; CI, confidence interval; aOR, adjusted odds ratio; Q1-Q5, quintiles 1-5; Ref., reference group.

Values in bold indicate statistical significance $(P<0 \cdot 05)$.

* All professional contact with children or young adult variables added simultaneously to M1.

$\dagger$ A separate model produced for each professional contact with children or young adult variable added to M1.

\$ Variables added to M1 in order of increasing statistical significance according to $P$ value from M3B, variables retained only if the lead to a statistically significant improvement in fit.

$\S \chi^{2}$ and associated $P$ value of the likelihood ratio test of improved fit compared to M1. 


\section{Adjusted associations between pertussis and all three categories of explanatory variables}

The final multivariable logistic regression model, considering variables from all three categories of potential predictors, contained both variables significantly associated with outcome in final models from the categorybased analysis: professional contact with children aged $<1$ year (aOR $0 \cdot 24,95 \%$ CI $0 \cdot 07-0 \cdot 76, P=0 \cdot 015$ ) and household contact with $\geqslant 1$ child aged $10-14$ years (aOR 2.66, 95\% CI 1.48-4.79, $P=0 \cdot 001$ ) (Table 5). The final model (M6) provided reasonable fit to the data $\left[\chi^{2}(99, n=418)=108 \cdot 49, P=0 \cdot 242\right]$. The point estimates of adjusted associations differed by only $4 \cdot 0 \%$ (professional contact with children aged $<1$ year) and $1.9 \%$ (household contact with $\geqslant 1$ child aged 10-14 years) from those of the category-only models.

\section{DISCUSSION}

This case-control study found two factors to be significantly associated with pertussis infection in adults and older teenagers in England: professional contact with children aged $<1$ year associated with reduced odds of pertussis, and sharing a household with $\geqslant 1$ child aged 10-14 years associated with increased odds of pertussis. Both of these factors were significant independent factors when contained within the same multivariable model, although both have a low prevalence in controls $(6 \cdot 8 \%$ reported professional contact with children aged $<1$ year, $11 \cdot 6 \%$ reported living with $\geqslant 1$ child aged 10-14 years) and will represent relatively small population attributable fractions. In addition to these factors, we observed a significantly higher odds of pertussis in the South West region of England, reflecting the high number of cases in this area at the time of the study [15].

Due to the higher than expected number of cases without a corresponding control, and to maximise statistical power, data were analysed using unconditional logistic regression with variables used for selection of controls (age and sex) included in all multivariable models. Any bias associated with the use of an unmatched analysis for a design including matching is towards a null effect, returning conservative estimates of risk [28]. As serological testing of controls was not undertaken, unrecognized or asymptomatic pertussis infection of controls cannot be excluded; it is certainly possible that individuals working with $<1$-year-olds may have developed mild or subclinical pertussis infection without having been aware. Although we did not collect pertussis vaccination history, and as such were unable to adjust for any potential confounding due to vaccination status, we are not aware of any obvious mechanism by which any confounding could occur; certainly, no programme has existed in England to offer pertussis booster vaccination to specific occupational groups or indeed to adults other than pregnant women (and only then offered from October 2012). There is therefore no obvious route through which pertussis vaccination could have been associated with either of the two factors found to be significantly associated with infection in this study.

Although HCWs have been implicated in transmission of pertussis within healthcare settings, we observed no significant association between working in a healthcare setting or direct patient contact with the odds of pertussis. We also found no similar association between working in education and social care. However, for all three exposures we cannot rule out misclassification of controls due to asymptomatic or mild pertussis infection. The lack of an association between working in healthcare and pertussis suggests that exposures for HCWs in our study do not differ significantly from that of the general population. Although a study in Brazil found that the seroprevalence of immunity to pertussis was higher in groups of HCWs working with children [22], a study in Germany supports the findings of our study [23].

While we did not observe a significant association between pertussis and working within a healthcare setting, we did observe a significantly reduced odds of pertussis for those individuals reporting professional contact with children aged $<1$ year. This association likely reflects frequent exposure to low levels of antigen that boost immunity to $B$. pertussis without causing a symptomatic infection [29]. Given that children aged $<1$ year consistently represent the age group with the highest incidence, including at the start of the 2011 pertussis increase in England [15], it is feasible that those working with children aged $<1$ year were more likely to have been immune-boosted by this mechanism prior to the increase in pertussis cases as the outbreak developed. No such association was observed for household contact with children aged $<1$ year, likely reflecting the relatively smaller number of individual children of this age group present within households compared with relevant professional settings.

There is good existing evidence to support the role of household members as the source of infant pertussis 
Table 5. Crude and adjusted associations between pertussis and occupational, professional contact with children or young adults, and household contact risk factors for adults and teenagers in England

\begin{tabular}{|c|c|c|c|c|c|c|c|c|c|}
\hline \multirow[b]{3}{*}{ Group } & \multirow[b]{3}{*}{ Variable } & \multirow[b]{3}{*}{ Category } & & & \multicolumn{5}{|c|}{ Multivariable models } \\
\hline & & & \multicolumn{2}{|c|}{ Univariable associations } & \multicolumn{2}{|l|}{ M1 } & & \multicolumn{2}{|l|}{ M6* } \\
\hline & & & uOR $(95 \% \mathrm{CI})$ & $P$ & $\mathrm{aOR}(95 \% \mathrm{CI})$ & $P$ & & $\mathrm{aOR}(95 \% \mathrm{CI})$ & $P$ \\
\hline \multirow[t]{11}{*}{ Demographics } & \multirow[t]{5}{*}{ Age quintiles } & Q1: $15-32$ years & $3 \cdot 20(1 \cdot 82-5 \cdot 95)$ & $<0 \cdot 001$ & $3 \cdot 56(1 \cdot 86-6 \cdot 79)$ & $<0 \cdot 001$ & & $3 \cdot 30(1 \cdot 68-6 \cdot 47)$ & $0 \cdot 001$ \\
\hline & & Q2: $33-43$ years & $2 \cdot 83(1 \cdot 52-5 \cdot 28)$ & $0 \cdot 001$ & $3 \cdot 23(1 \cdot 68-6 \cdot 22)$ & $<0 \cdot 001$ & & $2 \cdot 41(1 \cdot 22-4 \cdot 79)$ & $0 \cdot 012$ \\
\hline & & Q3: 44-51 years & $2 \cdot 24(1 \cdot 22-4 \cdot 13)$ & $0 \cdot 010$ & $2 \cdot 38(1 \cdot 25-4 \cdot 52)$ & $0 \cdot 008$ & & $1 \cdot 83(0 \cdot 93-3 \cdot 61)$ & $0 \cdot 081$ \\
\hline & & Q4: 52-61 years & $1 \cdot 42(0 \cdot 76-2 \cdot 68)$ & $0 \cdot 275$ & $1 \cdot 54(0 \cdot 80-2 \cdot 98)$ & $0 \cdot 204$ & & $1 \cdot 46(0 \cdot 74-2 \cdot 84)$ & $0 \cdot 272$ \\
\hline & & Q5: 62-87 years & Ref. & - & Ref. & - & & Ref. & - \\
\hline & \multirow[t]{2}{*}{ Sex } & Male & $1 \cdot 73(1 \cdot 15-2 \cdot 60)$ & $0 \cdot 008$ & $1 \cdot 84(1 \cdot 19-2 \cdot 83)$ & $0 \cdot 006$ & & $1 \cdot 70(1 \cdot 09-2 \cdot 66)$ & $0 \cdot 020$ \\
\hline & & Female & Ref. & - & Ref. & - & & Ref. & - \\
\hline & \multirow[t]{4}{*}{ Region } & London & $2 \cdot 49(1 \cdot 00-6 \cdot 22)$ & $0 \cdot 051$ & $1.98(0 \cdot 76-5 \cdot 11)$ & $0 \cdot 160$ & & $2 \cdot 09(0 \cdot 78-5 \cdot 58)$ & $0 \cdot 141$ \\
\hline & & South West & $2 \cdot 61(1 \cdot 24-5 \cdot 48)$ & $0 \cdot 011$ & $3 \cdot 14(1 \cdot 43-6 \cdot 94)$ & $0 \cdot 005$ & & $3 \cdot 03(1 \cdot 36-6 \cdot 73)$ & $0 \cdot 006$ \\
\hline & & West Midlands & $1 \cdot 45(0 \cdot 93-2 \cdot 27)$ & $0 \cdot 102$ & $1 \cdot 42(0 \cdot 89-2 \cdot 26)$ & $0 \cdot 141$ & & $1 \cdot 36(0 \cdot 84-2 \cdot 19)$ & $0 \cdot 209$ \\
\hline & & Yorkshire \& the Humber & Ref. & - & Ref. & - & & Ref. & - \\
\hline Travel & Recent travel & Yes & $0 \cdot 76(0 \cdot 50-1 \cdot 17)$ & $0 \cdot 222$ & $0 \cdot 67(0 \cdot 42-1 \cdot 07)$ & $0 \cdot 094$ & & $0 \cdot 68(0 \cdot 42-1 \cdot 10)$ & $0 \cdot 119$ \\
\hline \multirow[t]{4}{*}{ Occupation } & Social care & Yes & $1 \cdot 85(0 \cdot 63-5 \cdot 42)$ & $0 \cdot 262$ & - & - & & not included & - \\
\hline & Education & Yes & $0 \cdot 99(0 \cdot 62-1 \cdot 61)$ & $0 \cdot 982$ & - & - & & not included & - \\
\hline & Health & Yes & $1.02(0 \cdot 55-1 \cdot 88)$ & $0 \cdot 948$ & - & - & & not included & - \\
\hline & Patient contact & Yes & $1 \cdot 20(0 \cdot 62-2 \cdot 35)$ & 0.589 & - & - & & not included & - \\
\hline \multirow{9}{*}{\multicolumn{2}{|c|}{ Professional contact with children or young adults }} & $<1$ year & $0 \cdot 30(0 \cdot 11-0 \cdot 86)$ & $0 \cdot 025$ & - & - & - & $0 \cdot 24(0 \cdot 07-0 \cdot 76)$ & $0 \cdot 015$ \\
\hline & & Preschool (<5 years) & $0 \cdot 39(0 \cdot 16-0 \cdot 93)$ & $0 \cdot 034$ & - & - & - & not included & - \\
\hline & & Primary school (5-11 years) & $0 \cdot 76(0 \cdot 40-1 \cdot 45)$ & $0 \cdot 411$ & - & - & - & not included & - \\
\hline & & Young children $(<11$ years) & $0 \cdot 66(0 \cdot 36-1 \cdot 21)$ & $0 \cdot 183$ & - & - & - & not included & - \\
\hline & & Secondary school (11-16 years) & $0 \cdot 72(0 \cdot 35-1 \cdot 37)$ & 0.321 & - & - & - & not included & - \\
\hline & & College (16-18 years) & $0 \cdot 73(0 \cdot 40-1 \cdot 32)$ & $0 \cdot 300$ & - & - & - & not included & - \\
\hline & & University (18-21 years) & $0 \cdot 77(0 \cdot 43-1 \cdot 38)$ & $0 \cdot 371$ & - & - & - & not included & - \\
\hline & & Older children (11-21 years) & $0 \cdot 87(0 \cdot 53-1 \cdot 42)$ & $0 \cdot 569$ & - & - & - & not included & - \\
\hline & & Any age & $0 \cdot 86(0 \cdot 55-1 \cdot 34)$ & $0 \cdot 498$ & - & - & - & not included & - \\
\hline \multirow[t]{11}{*}{ Household contacts } & \multirow[t]{9}{*}{$\geqslant 1$ child or young adult } & $<1$ year & $3 \cdot 12(0 \cdot 86-11 \cdot 34)$ & $0 \cdot 084$ & - & - & & not included & - \\
\hline & & $1-4$ years & $0 \cdot 86(0 \cdot 42-1 \cdot 76$ & $0 \cdot 687$ & - & - & & not included & - \\
\hline & & $5-9$ years & $1 \cdot 38(0 \cdot 82-2 \cdot 13)$ & $0 \cdot 256$ & - & - & & not included & - \\
\hline & & Young children ( $\leqslant 9$ years) & $1 \cdot 32(0 \cdot 82-2 \cdot 13)$ & $0 \cdot 257$ & - & - & & not included & - \\
\hline & & $10-14$ years & $3 \cdot 25(1.92-5 \cdot 50)$ & $<0.001$ & - & - & & $2 \cdot 66(1 \cdot 48-4 \cdot 76)$ & $0 \cdot 001$ \\
\hline & & $15-19$ years & $1 \cdot 50(0 \cdot 93-2 \cdot 42)$ & $0 \cdot 094$ & - & - & & not included & - \\
\hline & & $20-21$ years & $1.98(0 \cdot 75-5 \cdot 25)$ & $0 \cdot 171$ & - & - & & not included & - \\
\hline & & Older children (10-21 years) & $2 \cdot 55(1 \cdot 68-3 \cdot 85)$ & $<0.001$ & - & - & & not included & - \\
\hline & & Any age $(0-21$ years $)$ & $1 \cdot 93(1 \cdot 31-2 \cdot 85)$ & $0 \cdot 001$ & - & - & & not included & - \\
\hline & \multirow[t]{2}{*}{ Household size } & $\leqslant 2$ & Ref. & - & - & - & & not included & - \\
\hline & & $\geqslant 3$ & $1.94(1 \cdot 31-2 \cdot 86)$ & 0.001 & - & - & & not included & - \\
\hline
\end{tabular}

uOR, Unadjusted odds ratio; CI, confidence interval; aOR, adjusted odds ratio; Q1-Q5, quintiles 1-5; Ref., reference group.

Values in bold indicate statistical significance $(P<0 \cdot 05)$.

* Variables added to M1 in order of increasing statistical significance according to $P$ value of univariable association, variables retained only if inclusion led to a statistically significant improvement in fit. 
[30-32]. This study provides additional evidence that contact with children aged 10-14 years in the home is a significant risk factor for pertussis in older children and adults. For cases of adult pertussis, it is known that their own children and work colleagues are the most likely sources of infection [17]. Our findings suggest that it is specifically children aged 10-14 years, where susceptibility may have developed through waning of immunity following childhood vaccination [7], that provide the greatest risk of household transmission of pertussis to older children and adults. Older children within the 10-14 years age group in this study may represent birth cohorts who received an aP booster vaccine and may be associated with more rapidly waning immunity compared with older birth cohorts vaccinated only with wP vaccines [33]. Certainly, at the start of the 2011 outbreak of pertussis in England there were higher than expected numbers of cases in teenagers and adults [34]. This study lacks the statistical power to investigate whether the risk of exposure to household contacts of specific ages within the $10-14$ years age group is consistent.

Although there is empirical and modelling evidence that booster vaccination of adolescents is effective for reducing the incidence of pertussis in adolescents [35], there is limited evidence of an indirect reduction of disease for infants [10], and no evidence of an indirect effect for older children and adults. Herd immunity following pertussis vaccination appears to be restricted to vaccinated cohorts only [35]. A study in Australia found evidence of indirect effects in young, unimmunized infants only when adolescent booster vaccination formed part of a broader catch-up of all students within a high-school setting [36]. However, assessing the impact of vaccination on the burden of illness for pertussis in older children and adults is problematic due to low case ascertainment rates and non-specific symptomatology.

The primary focus of the childhood pertussis vaccination programme is to prevent infant disease and evidence is emerging that adolescent vaccination does not provide indirect protection to infants. However, the results of this study highlight the importance of children aged 10-14 years in pertussis transmission to older adolescents and adults and support the need for further work to consider the potential public health benefit of reducing numbers of adult pertussis cases through the indirect effects of an adolescent booster vaccination. This may be particularly relevant now that the maternal vaccination programme has been shown to be highly effective at protecting young infants against disease.

\section{ACKNOWLEDGEMENTS}

We thank all study participants and the health protection and field epidemiology staff who contributed to this study. We also thank George Kafatos for commenting on the statistical analysis plan and the study steering committee for their contribution to planning.

This research received no specific grant from any funding agency, commercial or not-for-profit sectors.

\section{DECLARATION OF INTEREST}

None.

\section{REFERENCES}

1. Warfel JM, Edwards KM. Pertussis vaccines and the challenge of inducing durable immunity. Current Opinion in Immunology 2015; 35: 48-54.

2. van Hoek AJ, et al. The burden of disease and health care use among pertussis cases in school aged children and adults in England and Wales; a patient survey. PLOS ONE 2014; 9: e111807.

3. Schellekens J, von König C-HW, Gardner P. Pertussis sources of infection and routes of transmission in the vaccination era. Pediatric Infectious Disease Journal 2005; 24: S19-24.

4. Campbell $\mathbf{H}$, et al. Accelerating control of pertussis in England and Wales. Emerging Infectious Diseases 2012; 18: 38-47.

5. Miller E. Overview of recent clinical trials of acellular pertussis vaccines. Biologicals 1999; 27: 79-86.

6. Wendelboe AM, et al. Duration of immunity against pertussis after natural infection or vaccination. Pediatric Infectious Disease Journal 2005; 24: S58-61.

7. Tartof SY, et al. Waning immunity to pertussis following 5 doses of DTaP. Pediatrics 2013; 131: e1047-1052.

8. Clark TA. Changing pertussis epidemiology: everything old is new again. Journal of Infectious Diseases 2014; 209: 978-981.

9. Skoff TH, Martin SW. Impact of tetanus toxoid, reduced diphtheria toxoid, and acellular pertussis vaccinations on reported pertussis cases among those 11 to 18 years of age in an era of waning pertussis immunity: a follow-up analysis. JAMA Pediatrics 2016; 170: 453458.

10. World Health Organization. Pertussis vaccines: WHO position paper - August 2015. Weekly Epidemiological Record 2015; 90: 433-460.

11. Warfel JM, Zimmerman LI, Merkel TJ. Acellular pertussis vaccines protect against disease but fail to prevent infection and transmission in a nonhuman primate model. Proceedings of the National Academy of Sciences USA 2014; 111: 787-792. 
12. Amirthalingam G, et al. Effectiveness of maternal pertussis vaccination in England: an observational study. Lancet 2014; 384: 1521-1528.

13. Dabrera G, et al. A case-control study to estimate the effectiveness of maternal pertussis vaccination in protecting newborn infants in England and Wales, 20122013. Clinical Infectious Diseases 2015; 60: 333-337.

14. Tan T, Trindade E, Skowronski D. Epidemiology of pertussis. Pediatric Infectious Disease Journal 2005; 24: S10-18.

15. Public Health England. Laboratory confirmed pertussis in England: data to end-August 2014. Health Protection Report 2014; 8: 2-5.

16. Wiley KE, et al. Sources of pertussis infection in young infants: a review of key evidence informing targeting of the cocoon strategy. Vaccine 2013; 31: 618-625.

17. De Serres G, et al. Morbidity of pertussis in adolescents and adults. Journal of Infectious Disease 2000; 182: 174 179.

18. Pascual FB, et al. Outbreak of pertussis among healthcare workers in a hospital surgical unit. Infection Control and Hospital Epidemiology 2006; 27: 546-552.

19. Baugh V, McCarthy N. Outbreak of Bordetella pertussis among oncology nurse specialists. Occupational Medicine 2010; 60: 401-405.

20. Bassinet $\mathbf{L}$, et al. Nosocomial pertussis outbreak among adult patients and healthcare workers. Infection Control and Hospital Epidemiology 2004; 25: 995-997.

21. Wright SW, Decker MD, Edwards KM. Incidence of pertussis infection in healthcare workers. Infection Control and Hospital Epidemiology 1999; 20: 120-123.

22. Cunegundes KSA, et al. Bordetella pertussis infection in paediatric healthcare workers. Journal of Hospital Infection 2015; 90: 163-166.

23. Riffelmann M, et al. Antibodies to pertussis antigens in pediatric health care workers. Pediatric Infectious Disease Journal 2002; 21: 381-383.

24. Health Protection Agency. Public Health Management of Pertussis. London: Health Protection Agency, 2012.
25. Bechini A, et al. Acellular pertussis vaccine use in risk groups (adolescents, pregnant women, newborns and health care workers): a review of evidences and recommendations. Vaccine 2012; 30: 5179-5190.

26. Hope $\mathbf{K}$, et al. Pertussis vaccination in child care workers: room for improvement in coverage, policy and practice. BMC Pediatrics 2012; 12: 98.

27. Hosmer DW, Lemeshow S. Applied Logistic Regression. New York: John Wiley \& Sons, 2001.

28. Breslow NE. Statistics in epidemiology: the case-control study. Journal of the American Statistical Association 1996; 91: 14-28.

29. Lavine JS, King AA, Bjornstad ON. Natural immune boosting in pertussis dynamics and the potential for long-term vaccine failure. Proceedings of the National Academy of Sciences USA 2011; 108: 7259-7264.

30. Bisgard KM, et al. Infant pertussis. Pediatric Infectious Disease Journal 2004; 23: 985-989.

31. Wendelboe AM, et al. Transmission of Bordetella pertussis to young infants. Pediatric Infectious Disease Journal 2007; 26: 293-299.

32. de Greeff SC, et al. Pertussis disease burden in the household: how to protect young infants. Clinical Infectious Diseases 2010; 50: 1339-1345.

33. Sheridan SL, et al. Waning vaccine immunity in teenagers primed with whole cell and acellular pertussis vaccine: recent epidemiology. Expert Review of Vaccines 2014; 13: 1081-1106.

34. Health Protection Agency. Laboratory-confirmed cases of pertussis reported to the enhanced pertussis surveillance programme (England and Wales). Health Protection Report 2012; 6: 4-7.

35. Lavine JS, et al. Short-lived immunity against pertussis, age-specific routes of transmission, and the utility of a teenage booster vaccine. Vaccine 2012; 30: 544-551.

36. Quinn H, McIntyre P. The impact of adolescent pertussis immunization, 2004-2009: lessons from Australia. Bulletin of the World Health Organization 2011; 89: 666-674. 